\title{
A COMPARATIVE STUDY USING SHUFFLED COMPLEX EVOLUTION AND DIFFERENTIAL EVOLUTION APPLIED TO ROBOTIC MANIPULATOR DESIGN
}

\author{
M. Brandão ${ }^{1}$, J. L. Dorício ${ }^{2}$, F. S. Lobato ${ }^{3}$, S. F. P. Saramago ${ }^{4}$ \\ ${ }^{1}$ Department of Mathematics (FACIP), milena@pontal.ufu.br \\ 2 Department of Mathematics (FACIP), jldoricio@pontal.ufu.br \\ ${ }^{3}$ School of Chemical Engineering, fslobato@feq.ufu.br \\ ${ }^{4}$ College of Mathematics, saramago@ufu.br
}

Federal University of Uberlândia, Av. João Naves de Ávila, 2121, Uberlândia-MG, zip code 38400-902, Brazil.

\begin{abstract}
In last decades, evolutionary approaches has been used extensively and demonstrated to be robust and efficient global optimization methods for engineering system design. Among these techniques, the Shuffled Complex Evolution (SCE) and the Differential Evolution algorithm $(D E)$ are two good examples found in literature. DE differs from other evolutionary algorithms in the mutation and recombination phases. Unlike some meta-heuristic techniques such as genetic algorithms and evolutionary strategies, where perturbation occurs in accordance with a random quantity, DE uses weighted differences between solution vectors to perturb the population. In SCE a population of solutions is generated and partitioned into several sub-populations (called complexes). Each complex evolves independently using the DE algorithm for a set number of evolutions. The complexes are then shuffled thereby enabling exchange of information among them. If convergence is not reached, the population is again divided and a new set of evolutions for each new-found complex is carried out. In this work, is proposed a comparative study and a hybrid approach involving the SCE and the DE algorithms. The methodology proposed is applied to design of three-revolute (3R) manipulators using an optimization problem that takes into account the characteristics of the workspace. For this purpose, a multi-objective optimization problem is formulated to obtaining the optimal geometric parameters of robot. The maximum workspace volume, the maximum system stiffness and the optimum dexterity are considered as the multi-objective functions. The results show that the procedure represents a promising alternative for the type of problem presented above.
\end{abstract}

Keywords: Shuffled Complex Evolution, Differential Evolution, robotic manipulator design.

\section{INTRODUCTION}

Industrial robots and computer-aided systems, are the latest trend in the automation of fabrication processes, since the advances in the sensors field allow to develop more sophisticated tasks. The use of robots in the industry is wide since they accomplish tasks that are 
dangerous or monotonous for humans, this is the case of a industrial robot used for cleaning in electrical substations, which works in the high voltage area [35]. In robotic manipulators, a fundamental characteristic that must be taken into account in the dimensional design is the volume of their workspace. It is crucial to calculate the workspace and its boundaries with perfect precision, because they influence the dimensional design, the manipulator's positioning in the work environment, and its dexterity to execute tasks $[2,3,4]$.

In literature, several investigations have focused on the properties of the workspace of open chain robotics with the purpose of emphasizing its geometric and kinematic characteristics. Ceccarelli [7] presented an algebraic formulation to determine the workspace of revolution manipulators. Lanni et al. [15] investigated and solved the design of manipulators in the form of an optimization problem that takes into account the characteristics of the workspace. They applied two different numerical techniques: the first using sequential quadratic programming (SQP) and simulated annealing. Abdel-Malek et al. [1] proposed a generic formulation to determine voids in the workspace of serial manipulators. Other researches have focused on determining the workspace boundary and on detecting the presence of voids and singularities in the workspace. Saramago et al. [30] proposed a form of characterizing the workspace boundary, formulating a general analytic condition to deduce the existence of cusp points at the interior and exterior boundaries of the workspace. Ceccarelli and Lanni [8] presented a suitable formulation for the workspace that can be used in the design of manipulators, which was formulated as a multi-objective optimization problem using the workspace volume and robot dimensions as objective functions. Bergamaschi et al. [3,4] studied the design of manipulators with three-revolute joints (3R) using an optimization problem that takes into account the characteristics of the workspace. The optimization problem is formulated considering the workspace volume as the objective function. Constraints are added to guarantee the regularity of the envelope and force the workspace to occupy a pre-established area. In addition to the previously mentioned works, it has been proposed to solve mixed-integer linear program approximations of path planning problems directly in the workspace, where selected points on the links are used to represent the robot geometry as well as obstacle avoidance constraints $[6,13]$. The technique has been extended in [10] to incorporate state-dependent and time varying constraints.

Traditionally, this kind of problem has been treated by using either classical or deterministic optimization techniques. In recent years however, the use of non-deterministic techniques or the coupling of these techniques with classical ones, thus forming a hybrid methodology, became very popular due to the simplicity and robustness of evolutionary techniques. Among the most recent strategies based on population, stands the Shuffled Complex Evolution (SCE) and the Differential Evolution algorithm (DE) for solving optimization problems.

In this contribution, a multi-objective optimization problem is formulated to obtaining the optimal geometric parameters of robot. The maximum workspace volume, the maximum system stiffness and the optimum dexterity are considered as the multi-objective functions. To solve this problem, the the Shuffled Complex Evolution (SCE) and the Differential Evolution Algorithm (DE) are used. This work is organized as follows. Section 2 present the general aspects regarding the mathematical modeling of the robotic manipulator. Section 3 present 
a brief review about the multi-objective problems. A review dedicated to the SCE and the DE techniques are presented in Sections 4 and 5, respectively. The results and discussion are presented in Section 6. Finally, the conclusions are outlined in Section 7.

\section{MATHEMATICAL MODELING OF THE ROBOTIC MANIPULATOR}

The manipulators with three rotational joints with orthogonal axes as described in Fig. $1[28]$.

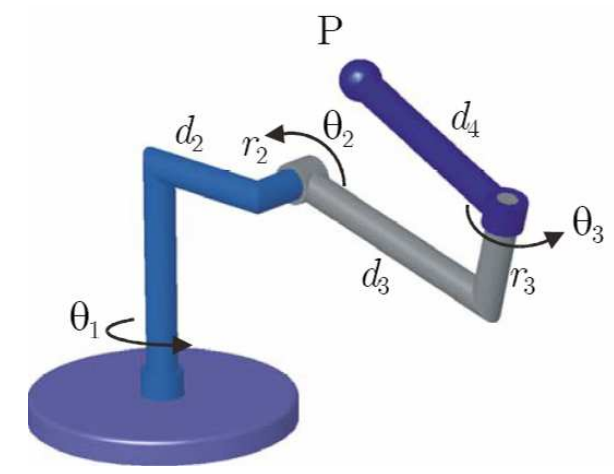

Figure 1. Manipulator with three rotational joints (3R) with orthogonal axes.

The study of this type of manipulator is done according to the Denavit-Hartenberg parameters: $d_{2}, d_{3}, d_{4}, r_{2}$, and $r_{3}$. To reduce the number of parameters and simplify the problem, will be considered $d_{2}=1$ and $r_{3}=0$. The joint variables are $\theta_{1}, \theta_{2}$ and $\theta_{3}$ which represent the input angles of the actuators. For this type of manipulator, the direct kinematic model is given in Eq. (1):

$$
\left\{\begin{array}{l}
x=\left[1+\left(d_{3}+d_{4} c_{3}\right) c_{2}\right] c_{1}-\left(r_{2}+d_{4} s_{3}\right) s_{1} \\
y=\left[1+\left(d_{3}+d_{4} c_{3}\right) c_{2}\right] s_{1}-\left(r_{2}+d_{4} s_{3}\right) c_{1} \\
z=-\left(d_{3}+d_{4} c_{3}\right) s_{2}
\end{array}\right.
$$

where $c_{i}=\cos \left(\theta_{i}\right)$ and $s_{i}=\sin \left(\theta_{i}\right)$, for $i=1,2,3$.

As mentioned earlier, a multi-objective optimization problem will be formulated to obtaining the optimal parameters of robot manipulator. The mathematical formulation for calculating the workspace volume, the system stiffness and the robot dexterity is shown below.

\subsection{Workspace of 3R Manipulators}

According to Bergamaschi et al. [3], the workspace $W$ is the set of all attainable points for a point $P$ of the end-effector when the joint variables sweep its definition interval entire. Point $P$ is usually chosen as the center of the end-effector, or the tip of a finger, or even the end of the manipulator itself. The first procedure to investigate the workspace is to vary the angles $\theta_{1}, \theta_{2}$ and $\theta_{3}$ in their interval of definition and to estimate the coordinates of point $P$ with respect to the manipulator base frame. The workspace of this robot is a solid of revolution. Thus, it is natural to imagine that the workspace is the result of rotation around the $\mathrm{z}$ axis of a radial plane section. 


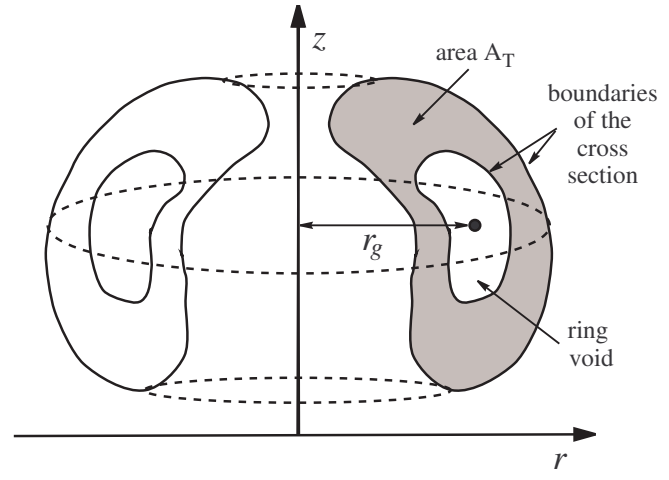

(a) Workspace volume.

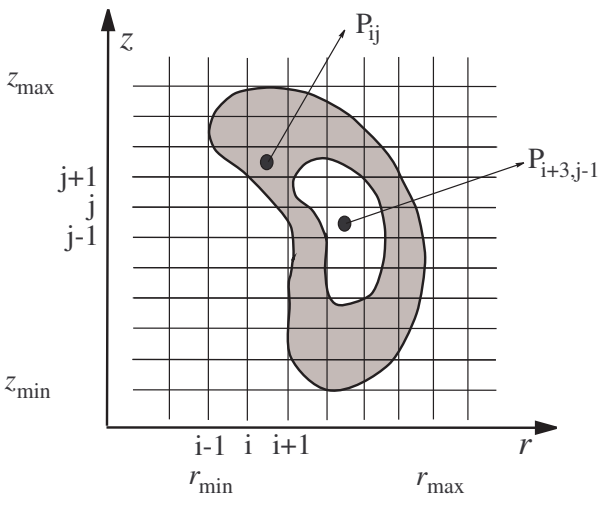

(b) Discretization of cross section.

Figure 2. A scheme for evaluating the workspace volume of $3 \mathrm{R}$ manipulators and discretization of cross section area by using a rectangular mesh.

The workspace of a three-revolute open chain manipulator can be given in the form of the radial reach $r$ and axial reach $z$ with respect to the base frame, according to Bergamaschi et al. [3]. For this representation, $r$ is the radial distance of a generic workspace point from the $z$-axis, and $z$ is the distance of this same point at the XY-plane (see, Fig. 2b). Thus, using Eq. (1), the parametric equations (of parameters $\theta_{2}$ and $\theta_{3}$ ) of the geometrical locus described by point $P$ on a radial plane are:

$$
r^{2}=x^{2}+y^{2}
$$

where $x, y$ and $z$ are given in Eq. (1).

The workspace volume $V$ can be evaluated by the Pappus-Guldin Theorem, using the following equation (see Fig. 2a):

$$
V=2 r_{g} A_{r}
$$

where $A_{r}$ is the cross section area, which is formed by the family of curves given by Eq. (1).

This research proposes numerical formulation to approximate the cross section area, through its discretization within a rectangular mesh. Initially, the extreme values of vectors $r$ and $z$ should be obtained as:

$$
r_{\text {min }}=\min r, \quad r_{\text {max }}=\max r, \quad z_{\text {min }}=\min z, \quad z_{\max }=\max z
$$

Adopting $n_{r}$ and $n_{z}$ as the number of intervals chosen for the discretization along the $r$ and $z$ axis, the sizes of the elementary areas of the mesh can be calculated:

$$
\begin{aligned}
& \Delta_{r}=\left(r_{\max }-r_{\min }\right) / n_{r} \\
& \Delta_{z}=\left(z_{\max }-z_{\min }\right) / n_{z}
\end{aligned}
$$

The $n_{r}$ and $n_{z}$ values must be adopted so that the sizes of the elementary areas $\left(\Delta_{r}\right.$ or $\left.\Delta_{z}\right)$ are at least $1 \%$ of the total distances considered in the discretization $\left(r_{\max }-r_{\min }\right.$ or 
$\left.z_{\max }-z_{\min }\right)$. Every point of the family of curves form the cross section of the workspace is calculated by Eq. (1). Using this equation, varying the values of $\theta_{2}$ and $\theta_{3}$ in the interval $[-\pi, \pi]$, it is possible to obtain the family of curves of the workspace. Given a certain point $(r, z)$, its position inside the discretization mesh is determined through the following index control:

$$
\begin{aligned}
& i=\operatorname{int}\left[\left(r-r_{\min }\right) / \Delta r\right]+1 \\
& j=\operatorname{int}\left[\left(z-z_{\min }\right) / \Delta z\right]+1
\end{aligned}
$$

where $i$ and $j$ are computed as integer numbers. As shown in Fig. 2b, the point of the mesh that belongs to the workspace is identified by $P_{i j}=1$, otherwise $P_{i j}=0$, which means:

$$
P_{i j}=0, \text { if } P_{i j} \notin W(P) \text { or } 1, \text { if } P_{i j} \in W(P)
$$

where $W(P)$ indicates workspace region.

In this way, the total area is obtained by the sum of every elementary areas of the mesh that are totally or partially contained in the cross section. In Eq. (9), it is observed that only the points that belong to the workspace contribute to the calculation of the area $A_{T}$. The coordinate $r_{g}$ of the center of the mass is calculated considering the sum of the center of the mass of each elementary area, divided by the total area, using the following equation:

$$
\begin{gathered}
A_{T}=\sum_{i=1}^{i_{\max }} \sum_{j=1}^{j_{\max }}\left(P_{i j} \Delta r \Delta z\right) \\
r_{g}=\left(\sum_{i=1}^{i_{\max }} \sum_{j=1}^{j_{\max }}\left(P_{i j} \Delta r \Delta z\right)\left((i-1) \Delta r+(\Delta r / 2)+r_{\min }\right)\right) / A_{T}
\end{gathered}
$$

Finally, after the calculation of the cross section area and the coordinate of the center of the mass, given by Eqs. (10) and (11), the workspace volume of the manipulator can be evaluated by using Eq. (3).

\subsection{System Stiffness}

From the mechanics viewpoint, the stiffness is the measurement of the ability of a body or structure to resist deformation due to the action of external forces. The stiffness of a serial mechanism at a given point of its workspace can be characterized by its stiffness matrix. This matrix relates the forces and torques applied at the gripper link in Cartesian space to the corresponding linear and angular Cartesian displacements.

Two main methods have been used to establish mechanism stiffness models. The first one is called matrix structural analysis, which models structures as a combination of elements and nodes. The second method relies on the calculation of the serial mechanism's Jacobian matrix which is adopted in this work. Matrix $J$ is usually termed Jacobian matrix which is described in Eq. (12). By considering the case in that $d_{2}=1$, its determinant is calculated by using the Eq. (13). 


$$
\begin{gathered}
{[J]=\left(\begin{array}{ccc}
-\sin \theta_{3} \cos \theta_{2} d_{4}-\cos \theta_{2} r_{2} & 0 & -\sin \theta_{3} d_{4} \\
\sin \theta_{3} \sin \theta_{2} d_{4}+\sin \theta_{2} r_{2} & d_{3}+\cos \theta_{3} d_{4} & 0 \\
\cos \theta_{2} d_{3}+\cos \theta_{2} \cos \theta_{3} d_{4}+d_{2} & 0 & \cos \theta_{3} d_{4}
\end{array}\right)} \\
\operatorname{det}(J)=d_{4}\left(d_{3}+d_{4} \cos \theta_{3}\right)\left[d_{2} \sin \theta_{3}+\left(d_{3} \sin \theta_{3}+\left(d_{3} \sin \theta_{3}-r_{2} \cos \theta_{3}\right) \cos \theta_{2}\right)\right]
\end{gathered}
$$

The stiffness matrix of the mechanism in the Cartesian space is then given by the Eq. (14), where $K_{j}$ is the joint stiffness matrix of the mechanism, with $K_{j}=\left[k_{1}, k_{2}, k_{3}\right]$. In this case, each actuators of the mechanism is modeled as an elastic component. $k_{i}$ is a scalar representing the joint stiffness of each actuator, which is modeled as linear spring:

$$
K_{C}=[J]^{T} K_{j}[J]
$$

Particularly, in the case for which all the actuators have the same stiffness, e.g., $k=$ $k_{1}=k_{2}=k_{3}$, then Eq. (14) will be reduced to:

$$
K_{C}=k[J]^{T}[J]
$$

Furthermore, the diagonal elements of the stiffness matrix are used as the system stiffness value. These elements represent the pure stiffness in each direction, and they reflect the rigidity of machine tools more clearly and directly. The objective function for system stiffness optimization can be written as Eq. (16). In this case, the stiffness index $S$ can be maximized:

$$
S=K_{11}+K_{22}+K_{33}
$$

\subsection{Dexterity}

The condition number of the Jacobian matrix will be used as a measure of dexterity indices for the 3R manipulator. By using the spectral norm, these indices will be described as.

$$
\operatorname{Cond}(J)=\left|\lambda_{\max }(J) / \lambda_{\min }(J)\right|
$$

where $\lambda_{\max }$ and $\lambda_{\min }$ means the maximum and minimum singular values of Jacobian matrix $J$, respectively. Regarding the computing time of optimization process, this expression is selected as the objective function for the optimization of dexterity. The value of $\operatorname{Cond}(J)$, which is directly related to singular values of Jacobian matrix, is between 1 and positive infinity. All the singular values of the Jacobian matrix will be the same and the manipulator is isotropic if $\operatorname{Cond}(J)$ is equal to 1 . While $\operatorname{Cond}(J)$ is prone to be positive infinity it means that the Jacobian matrix is singular. Therefore, for the optimization of dexterity, the condition number must to be minimized.

\section{MULTI-OBJECTIVE OPTIMIZATION}

In many situations, multiple objective functions must be minimized and/or maximized simultaneously, and in this case, the problem optimization is called problem multi-purpose (or multi-criteria). 
The objective function of problem can be rewritten as a vector $f(x)$, whose components are $k$ functions objectives that wish to maximize and/or minimize. The vector function may be written as:

$$
\text { Maximize or Minimize } f(x)=\left[f_{1}(x), f_{2}(x), \cdots, f_{k}(x)\right]^{T}
$$

There are several methods to solve this problem [29]. In this study will be used Global Criterion Method (GCM) and the Weighting Objectives Method (WOM). These techniques consist of transforming the vector of objective functions in a scalar function.

In the GCM, the optimal solution is a vector of design variables that minimizes a global criterion. The function that describes this criterion should be defined by the designer in order to obtain a solution as close as possible the ideal solution. This global function can be written as a family of metrics- $L_{P}$, defined as:

$$
L_{p}=\left[\sum_{i=1}^{k}\left|f_{i}^{0}-f_{i}(x)\right|^{s}\right]^{1 / s}, \quad 1 \leq s \leq \infty
$$

Instead of working with distance in an absolute sense, it is recommended to use relative distances, so the equation (19) can be rewritten as:

$$
L_{p}=\left[\sum_{i=1}^{k}\left|\frac{f_{i}^{0}-f_{i}(x)}{f_{i}^{0}}\right|^{s}\right]^{1 / s}, \quad 1 \leq s \leq \infty
$$

Using the relative metric $L_{2 R}$ have been given scalar functions, respectively, by:

$$
L_{2 R}=\left(\left(\frac{f_{1}^{0}-f_{1}(x)}{f_{1}^{0}}\right)^{2}+\cdots+\left(\frac{f_{k}^{0}-f_{k}(x)}{f_{k}^{0}}\right)^{2}\right)^{1 / 2}
$$

Thus, in Equation (21), the vector function was normalized by using the concept of ideal $f_{k}^{0}$. This solution is determined separately obtaining the optimal feasible, for all objective functions. In other words:

$$
f_{k}^{0}=\min f_{k}(x), \quad k=1, \cdots, K
$$

In the other hand, the WOM converts the multi-objective problem of optimizing the vector $f(x)$ into a scalar problem by building a weighted sum of all the objectives:

$$
f(x)=\sum_{i=1}^{k} w_{i} f_{i}(x) r_{i}
$$

with

$$
\sum_{i=1}^{k} w_{i}=1
$$

where $r_{i}$ are constant multipliers, $w_{i}>0$ are the weighting coefficients that represent the relative importance of each criterion. Objective weighting is obviously the most usual substitute 
model for vector optimization problems. The trouble here is attaching weighting coefficients to each of the objectives. The weighting coefficients do not necessarily correspond directly to the relative importance of the objectives or allow trade-offs between the objectives to be expressed. For the numerical methods for seeking the optimum of Eq. (23) so that $w_{i}$ can reflect closely the importance of objectives, all the functions should be expressed in units of approximately the same numerical values.

The best results are usually obtained if $r_{i}=1 / f_{i}^{0}$, where $f_{i}^{0}$ represents the ideal solution for the problem.

\section{SHUFFLED COMPLEX EVOLUTION ALGORITHM}

According to Duan [11] and Liong and Atiquzzaman [16], SCE works on the basis of four concepts: (1) combination of deterministic and probabilistic approaches; (2) systematic evolution of a complex of points; (3) competitive evolution; and (4) complex shuffling. The algorithm begins with a randomly selected population of points from the feasible space. The points are sorted in order of increasing criterion value so that the first point represents the smallest function value and the last point represents the largest function value. The randomly generated initial population is partitioned into several complexes. Each complex is allowed to evolve independently to search the feasible domain in different directions. Each individual point in a complex has the potential to participate in the process of reproducing new points. From each complex, some points are selected to form a subcomplex, where the modified Nelder and Mead Simplex Method (NMSM) [27])is applied for global improvement. The points of higher fitness values have higher chance of getting selected to generate offspring. The NMSM performs reflection and inside contraction step to get a better fit point. This new offspring replaces the point with the worst performance in the simplex. The points in the evolved complexes are then pooled together and is sorted again, shuffled, and finally reassigned to new complexes to enable information sharing. This process is repeated until some stopping criteria are satisfied.

The control parameters in SCE are the following: number of complexes, minimum number of complexes required (if the number of complexes is allowed to reduce as the optimization proceeds), number of points in each complexes in the initial population, number of points in each sub-complex, number of offspring generated by each sub-complex, and number of evolution steps allowed for each complex before complex shuffling. SCE has two stopping criteria checked at each generation. The evaluation will stop when one of the following criteria is arrived first: $(i)$ the relative change in the objective function values within the last $k$, say 10-15 shuffling loops is less than a pre-specified tolerance; (ii) the maximum user-specified number of function evaluations is reached.

SCE has been applied in calibrating rainfall-runoff models [12,14,26], optimal design ow water distribution network [16], and multi-objective auto-calibration for semi-distributed water quality models [34]. 


\section{DIFFERENTIAL EVOLUTION ALGORITHM}

Differential Evolution (DE) is an optimization technique that belongs to the family of evolutionary computation, which differs from other evolutionary algorithms in the mutation and recombination schemes. DE executes its mutation operation by adding a weighted difference vector between two individuals to a third individual. Then, the mutated individuals will perform discrete crossover and greedy selection with the corresponding individuals from the last generation to produce the offspring.

The control parameters in DE are the following: $N$, the population size, $C R$, the crossover constant and, $F$, the weight applied to random differential (perturbation rate). According to Storn and Price [31,32], $N$ should be about 5 to 10 times the dimension (number of parameters in a vector) of the problem, $F$ and $C R$ should be in the range 0.4 to 1.0. The structure of the basic Differential Evolution Algorithm is shown in Fig. 3 [31,32].

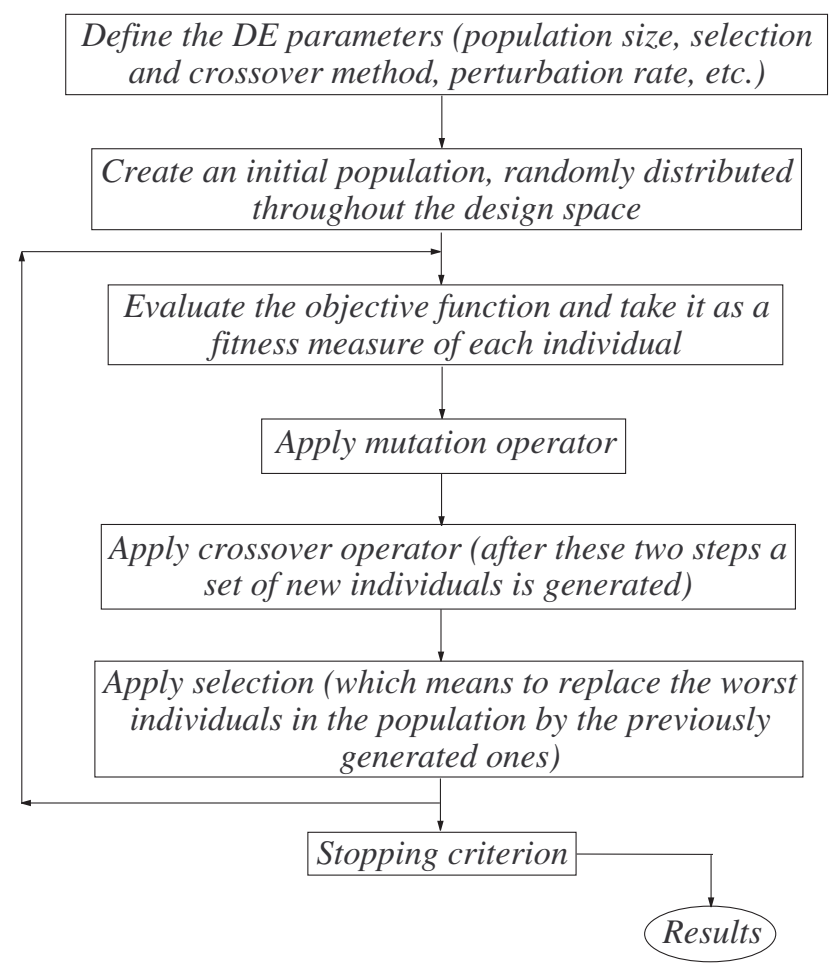

Figure 3. Differential Evolution Algorithm.

Storn et al. [31] proposed various mutation schemes for the generation of new vectors (candidate solutions) by combining the vectors that are randomly chosen from the current population as shown:

- $\mathrm{rand} / 1: x=x_{r 1}+F\left(x_{r 2}-x_{r 3}\right)$

- $\mathrm{rand} / 2: x=x_{r 1}+F\left(x_{r 2}-x_{r 3}+x_{r 4}-x_{r 5}\right)$

- best/1: $x=x_{\text {best }}+F\left(x_{r 2}-x_{r 3}\right)$

- best/2: $x=x_{\text {best }}+F\left(x_{r 2}-x_{r 3}+x_{r 4}-x_{r 5}\right)$ 
- $\mathrm{rand} / \mathrm{best} / 1: x=x_{r 1}+F\left(x_{\text {best }}-x_{r 1}+x_{r 1}-x_{r 2}\right)$

- $\mathrm{rand} / \mathrm{best} / 2: x=x_{r 1}+F\left(x_{\text {best }}-x_{r 1}\right)+F\left(x_{r 1}-x_{r 2}+x_{r 3}-x_{r 4}\right)$

DE has been successfully tested in various fields, such as: solution of multi-objective optimal control problems with index fluctuation applied to fermentation process [17], digital filter design [33], multi-objective optimization of mechanical structures [18], solution of inverse radiative transfer problems in two-layer participating media [19], estimation of drying parameters in rotary dryers [20], apparent thermal diffusivity estimation of the drying of fruits [25], Gibbs free energy minimization in a real system [21], estimation of space-dependent single scattering albedo in radiative transfer problems $[22,23,24]$, design of fractional order PID controllers [5], and other applications [9,31,32].

\section{NUMERICAL SIMULATION}

The objective of the proposed manipulator design procedure, considering the workspace volume $(V)$, the system stiffness $(S)$ and the manipulator dexterity $(D)$, is the dimensional synthesis of $3 \mathrm{R}$ orthogonal robot.

The multi-objective optimization problem is defined as:

$$
\max f(x)=[V-\operatorname{Cond}(J) S]
$$

subject to $0 \leq x_{i} \leq 3, i=1,2,4$, where the geometric parameters are design variables given by $x=\left[\begin{array}{llll}d_{3} & d_{4} & r_{2} & r_{3}\end{array}\right]^{T}$. The volume workspace is given by Eq. (3), the system stiffness is given by Eq. (16). For the optimization of dexterity, the condition number $(\operatorname{Cond}(J))$, given by Eq. (17), must to be minimized.

In order to evaluate the performance of both the techniques proposed above (SCE and DE), the following points should be emphasized:

- the computational codes of the DE and SCE were developed in Matlab ${ }^{\circledR}$ by the authors;

- DE parameters: population size (9), perturbation rate (0.8), crossover probability (0.6), and DE/best/1/exp strategy;

- SCE parameters: population size (9 individuals in each complex), the starting point is the midpoint of the lateral limits of the variables, namely $x_{0}=\left[\begin{array}{llll}1.45 & 1.45 & 1.45 & 1.45\end{array}\right]$, perturbation rate (0.8), crossover probability $(0.6)$;

- the stopping criterion used by DE was the maximum number of iterations (100). The stopping criterion used by SCE was the normalized geometric range of the parameters less than 0.0001;

- DE was executed 20 times to obtain the average values presented in tables;

- robot parameters considered: $d_{2}=1, d_{3}=x(1), d_{4}=x(2), r_{2}=x(3), r_{3}=x(4), a_{2}=$ $-\pi / 2, a_{3}=\pi / 2$, the size step for calculating the Jacobian: 0.03 and mesh: $50 \times 50$; 
- all simulations were solved by using a computer Intel ${ }^{\circledR}$ Core $^{T M}$ i5-430M Processor and 6 GB of RAM.

In the following tables are summarized the optimal values found by using the Differential Evolution and Shuffled Complex Evolution (with 2, 4 e 8 complexes) optimization methods.

Table 1. Optimal values considering the Global Criterion Method $\left(L_{2 R}\right.$ Metric).

\begin{tabular}{|c|c|c|c|c|c|c|}
\hline Algorithm & Volume [v.u.] & Dexterity & Stiffness [s.u.] & 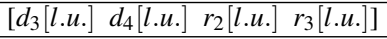 & Time $[\mathrm{h}]$ & $N_{\text {eval }}$ \\
\hline DE & $\begin{array}{c}1902.70 \\
1903.30^{*} \\
0.74^{* *} \\
\end{array}$ & $\begin{array}{c}1.04 \\
1.04^{*} \\
0.01^{* *} \\
\end{array}$ & $\begin{array}{c}105.68 \\
105.68^{*} \\
0.01^{* *}\end{array}$ & $\begin{array}{c}{\left[\begin{array}{llll}3 & 3 & 3 & 0.1\end{array}\right]} \\
{\left[\begin{array}{llll}3.00 & 3.00 & 3.00 & 0.11\end{array}\right]^{*}} \\
{\left[\begin{array}{llll}0 & 0 & 0 & 0.01\end{array}\right]^{* *}}\end{array}$ & 8.03 & 150 \\
\hline $\begin{array}{cc}\text { ECE-DE } \\
2 \text { complexes }\end{array}$ & $\begin{array}{c}1905.10 \\
1844.85^{*} \\
132.71^{* *}\end{array}$ & $\begin{array}{c}1.14 \\
1.14^{*} \\
0.12^{* *}\end{array}$ & $\begin{array}{c}105.90 \\
103.51^{*} \\
5.19^{* *}\end{array}$ & 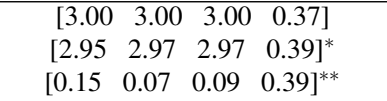 & 3.05 & 1462 \\
\hline $\begin{array}{c}\text { ECE-DE } \\
4 \text { complexes }\end{array}$ & $\begin{array}{c}1908.20 \\
1805.18^{*} \\
137.30^{* *}\end{array}$ & $\begin{array}{c}1.11 \\
1.13^{*} \\
0.13^{* *}\end{array}$ & $\begin{array}{c}105.82 \\
102.17^{*} \\
5.13^{* *}\end{array}$ & 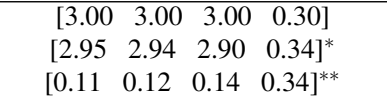 & 7.24 & 3468 \\
\hline $\begin{array}{c}\text { ECE-DE } \\
8 \text { complexes }\end{array}$ & $\begin{array}{c}1903.40 \\
1832.20^{*} \\
142.31^{* *}\end{array}$ & $\begin{array}{c}1.08 \\
1.10^{*} \\
0.06^{* *}\end{array}$ & $\begin{array}{c}105.71 \\
103.23^{*} \\
5.15^{* *}\end{array}$ & 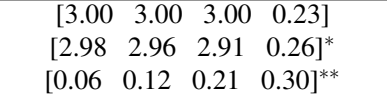 & 14.23 & 6800 \\
\hline
\end{tabular}

Table 2. Optimal values considering the Weighting Objectives Method.

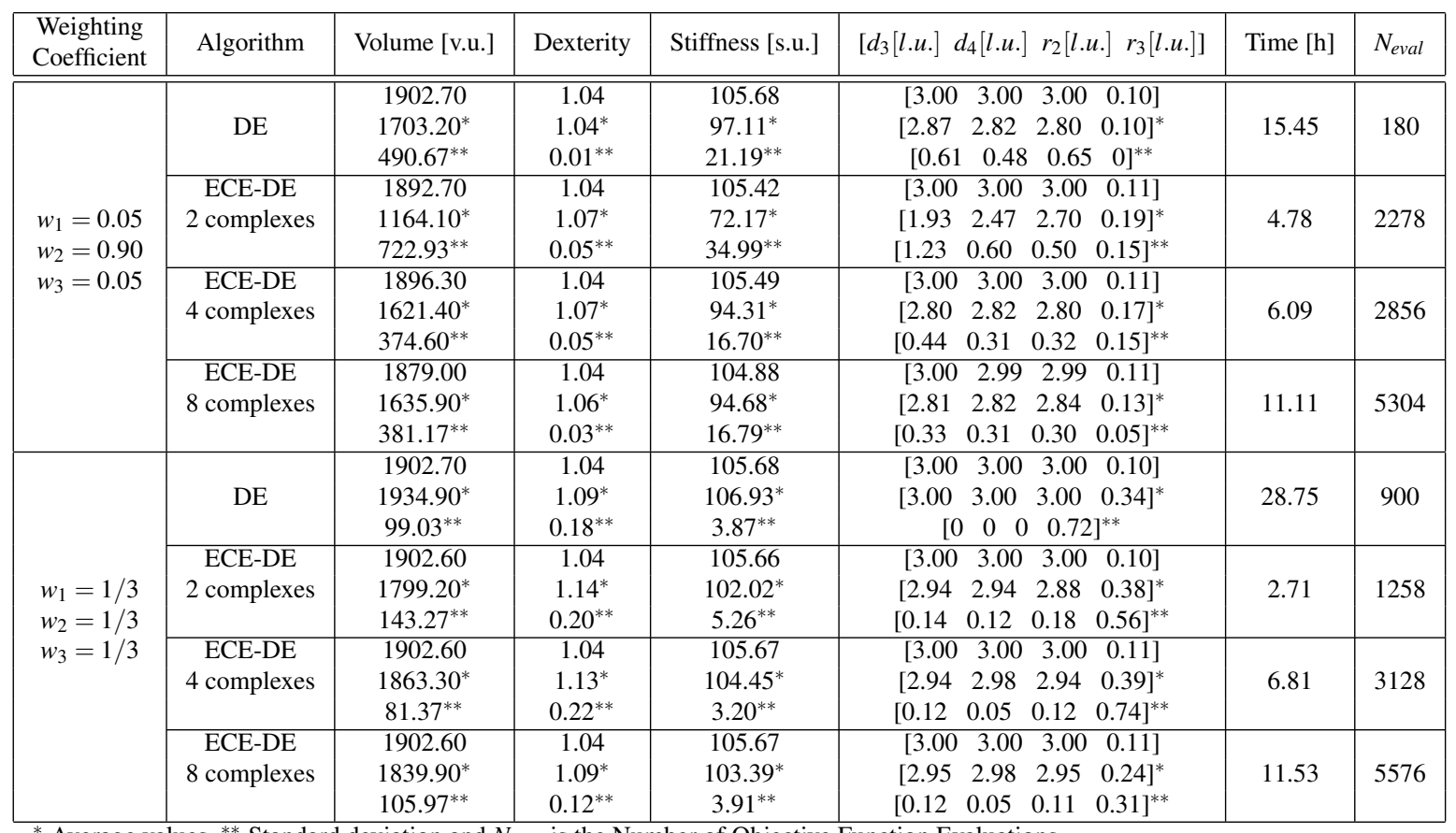

${ }^{*}$ Average values, ${ }^{* *}$ Standard deviation and $N_{\text {eval }}$ is the Number of Objective Function Evaluations.

It is worth noting that the optimal results present in Tab. (2) are strongly dependent on the weighting coefficients. This table shows the results of the case in which the optimization of the dexterity is prioritized $\left(w_{2}=0.9\right)$.

Considering the ideal values of the workspace volume, dexterity and system stiffness are, $V_{\text {ideal }}=2382.7412$ [v.u.], $D_{\text {ideal }}=1.0256$ and $S_{\text {ideal }}=125.0769$ [s.u.], respectively, one can concluded that the methods were effective in solving the problem since the results approach the ideals. Comparing the results obtained with DE and SCE, it is possible to observe that are 
very similar. However, for the studied problem, the SCE method reached the optimum faster. This analysis should be very careful, these results do not mean that this method is always the best.

Figures (4), (5) and (6) shows the graph of the cross-sectional area of the workspace of the robot manipulator: (1) DE, (2) SCE with 2 complexes, (3) SCE with 4 complexes e (4) SCE with 8 complexes, considering the Global Criterion Method and the Weighting Objectives Method, respectively.
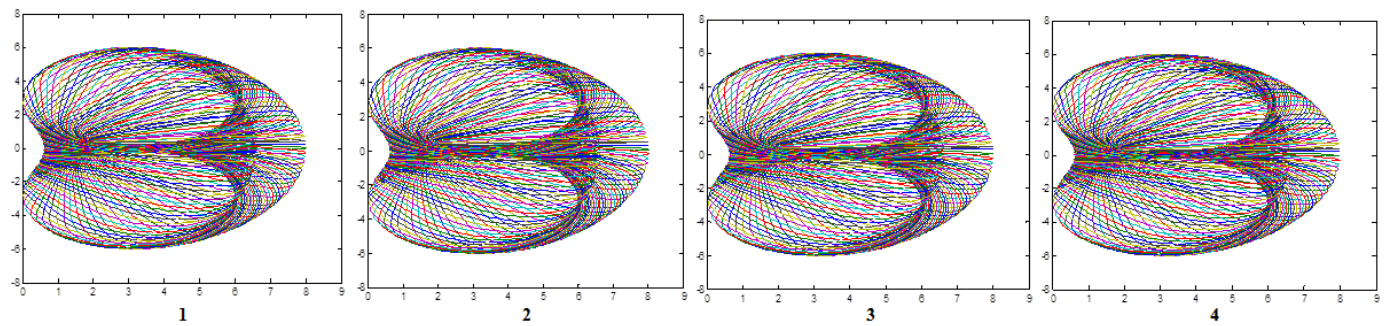

Figure 4. Cross-sectional area of the workspace of the robot manipulator considering the Global Criterion Method: (1) DE, (2) SCE with 2 complexes, (3) SCE with 4 complexes e (4) SCE with 8 complexes.
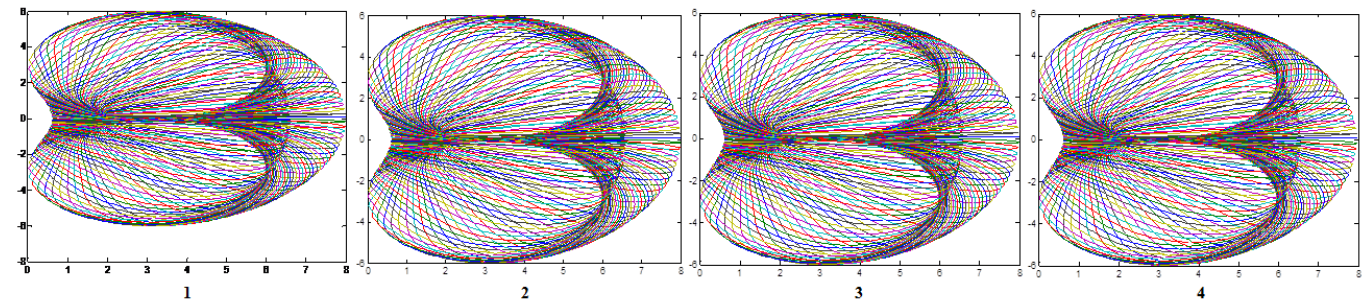

Figure 5. Cross-sectional area of the workspace of the robot manipulator considering the Weighting Objectives Method for $w_{1}=0.05, w_{2}=0.90$ and $w_{3}=0.05$ : (1) DE, (2) SCE with 2 complexes, (3) SCE with 4 complexes e (4) SCE with 8 complexes.


Figure 6. Cross-sectional area of the workspace of the robot manipulator considering the Weighting Objectives Method for $w_{1}=1 / 3, w_{2}=1 / 3$ and $w_{3}=1 / 3$ : (1) DE, (2) SCE with 2 complexes, (3) SCE with 4 complexes e (4) SCE with 8 complexes. 


\section{CONCLUSIONS}

In this contribution, two different evolutionary techniques, the Differential Evolution algorithm and the Shuffled Complex Evolution algorithm, were used to solve a multi-objective problem. A optimal manipulator design was presented to illustrate the methodology studied here. Successful numerical applications have demonstrated the efficiency of these techniques. The preliminary results seem the indicated that the SCE is faster to achieve the optimal solution. The results are improved when increasing the number of complexes, but it means to increase the computational time.

In the future works, the aim is to combine the best characteristics of the Differential Evolution and Shuffled Complex Evolution algorithms and implement them in parallel, as well, each set of the ECE method will evolve in a different processor and at the same time, following the principle that large problems can generally be divided into smaller problems, which are then solved concurrently (in parallel).

The idea of dividing tasks across multiple processors programs is old but only recently has become feasible due to the rapid advancement of hardware and software. The machines have evolved greatly in speed and with the emergence of machines with multiple processors, the communication speed is showing a great evolution allows these processors to exchange information quickly, and programs that enable this communication are showing a great increase in efficiency. Thus, it is intended to reduce the processing time for the implementation of improved algorithm to enable its application to complex problems.

\section{Acknowledgements}

The authors acknowledge the financial support provided by FAPEMIG (Fundação de Amparo à Pesquisa de Minas Gerais) and CAPES.

\section{REFERENCES}

[1] Abdel-Malek K., Yeh H. -J., Othman S., "Understanding Voids in the Workspace of Serial Robot Manipulators", Proceedings Pf 23rd ASME, Design Engineering Technical Conference, Baltimore, Maryland, 2000.

[2] Beer F. P., Johnston Jr. E. R., "Vector Mechanics for Engineers: Statics and Dynamics", Mac Graw Hill, third edition, New York, USA, 1977.

[3] Bergamaschi P. R., Nogueira A. C., Saramago S. F. P, "Design and Optimization of 3R Manipulators using the Workspace Features", Applied Mathematics and Computation, 172, 439-463, 2006.

[4] Bergamaschi P. R., Saramago S. F. P, Coelho, L. S., "Comparative Study of SQP and Metaheuristics for Robotic Manipulator Design", Applied Mathematics and Computation, 58, 1396-1412, 2008.

[5] Biswas A., Das S., Abraham A., Dasgupta S., "Design of Fractional order PID Controllers with an Improved Differential Evolution", Engineering Applications of Artificial Intelligence, Elsevier Science, 22, 2, 343-350, 2009. 
[6] Blackmore L., Williams B., "Optimal Manipulator Path Planning with Obstacles using Disjunctive Programming", Proc. of the American Control Conference, 32003202, 2006.

[7] Ceccarelli M., "A Formulation for the Workspace Boundary of General N-Revolute Manipulators”, IFToMM Journal of Mechanism and Machine Theory, 31, 5, 637646, 1996.

[8] Ceccarelli M., Lanni C., "A Multi-objective Optimum Design of General 3R Manipulators for Prescribed Workspace Limits, Mechanism and Machine Theory, 39, 119-132, 2004.

[9] Das S., Suganthan P. N., "Differential Evolution: A Survey of the State-of-the-Art", IEEE Trans. on Evolutionary Computation, DOI: 10.1109/TEVC.2010.2059031, 2011.

[10] Ding H., Zhou M., Stursberg O., "Optimal Path Planning in the Workspace for Articulated Robots using Mixed Integer Programming", IEEE/RSJ Int. Conf. on Intell. Robots and Syst., 5770-5775, 2009.

[11] Duan Q., "A Global Optimization Strategy for Efficient and Effective Calibration of Hydrologic Models", Dissertation, University of Arizona, USA.

[12] Franchini M., Galeati G., Berra S., "Global Optimisation Techniques for the Calibration of Conceptual Rainfall-Runoff Models", Journal of Hydrologic Science, 43, 3, 443-458, 1998.

[13] Gupta K. C., Roth B., "Design Considerations for Manipulator Workspace”, Journal of Mechanical Design, 104, 704-711, 1982.

[14] Kuczera G., "Efficient Subspace Probabilistic Parameter Optimization for Catchment Models", Water Resources Research, 33, 177-185, 1997.

[15] Lanni C., Saramago S.F.P., Ceccarelli M., "Optimal Design of 3R Manipulators using Classical Techniques and Simulated annealing", Revista Brasileira de Cincias Mecnicas, Brazil, 24, 4, 293-301, 2002.

[16] Liong S. Y., Atiquzzaman M., "Optimal Design ow Water Distribution Network using Shuffled Complex Evolution", Journal of The Institution of Engineers, Singapore, 44, 1, 2004.

[17] Lobato F. S., Oliveira-Lopes L. C., Murata V. V., Steffen Jr. V., "Solution of Multiobjective Optimal Control Problems with Index Fluctuation using Differential Evolution". 6th Brazilian Conference on Dynamics, Control and Applications - DINCON, 2007.

[18] Lobato F. S., Steffen Jr. V., "Engineering System Design with Multi-objective Differential Evolution", 19th International Congress of Mechanical Engineering - COBEM 2007.

[19] Lobato F. S., Arruda E. B., Barrozo M. A. S., Steffen Jr. V., "Estimation of Drying Parameters in Rotary Dryers using Differential Evolution". Journal of Physics Conference Series, 135, 1-8, 2008.

[20] Lobato F. S., Steffen Jr. V., Silva Neto A. J.. "Solution of Inverse Radiative Transfer Problems in Two-layer Participating Media with Differential Evolution", EngOpt 2008 - International Conference on Engineering Optimization, Rio de Janeiro Brasil, 2008. 
[21] Lobato F. S., Figueira C. E., Soares R. R., Steffen Jr. V., “A Comparative Study of Gibbs Free Energy Minimization in a Real System Using Heuristic Methods". Computer-Aided Chemical Engineering, 27, 1059-1064, 2009.

[22] Lobato F. S., Steffen Jr. V., Silva Neto A. J., "Estimation of Space-dependent Single Scattering Albedo in Radiative Transfer Problems", Inverse Problems, Design and Optimization Symposium, João Pessoa, Brazil, 25-27, 2010.

[23] Lobato F. S., Steffen Jr. V., Silva Neto A. J., "A Comparative Study of the Application of Differential Evolution and Simulated Annealing in Inverse Radiative Transfer Problems". Journal of the Brazilian Society of Mechanical Sciences and Engineering, XXXII, 518-526, 2010.

[24] Lobato F. S., Steffen Jr. V., Silva Neto A. J., "Self-Adaptive Differential Evolution Based on the Concept of Population Diversity Applied to Simultaneous Estimation of Anisotropic Scattering Phase Function, Albedo and Optical Thickness". Computer Modeling in Engineering \& Sciences, 1, 1-17, 2010.

[25] Mariani V. C., Lima A. G. B., Coelho L. S., "Apparent Thermal Diffusivity Estimation of the Banana During Drying using Inverse Method", Journal of Food Engineering, 85, 569-579, 2008.

[26] Muttil N., Liong S. Y., "A Superior Exploration-Exploitation Balance in Shuffled Complex Evolution", Journal of Hydraulic Engineering, ASCE, 130, 12, 12021205, 2004.

[27] Nelder J. A., Mead R., "A Simplex Method for Function Minimization”, Computer Journal, 7, 308-313, 1965.

[28] Oliveira G. T. S., Nogueira, A. C., Saramago, S. F. P., "Use of the Grobner Basis in the Study of Manipulators Topology", Proceedings of the 20th International Congress of Mechanical Engineering, Gramado, 1-10, 2009.

[29] Oliveira L. S., Saramago, S. F. P.’Multiobjective Optimization Techniques Applied to Engineering Problems", Journal of the Brazilian Society of Mechanical Sciences and Engineering, XXXII, 94-104, 2010.

[30] Saramago, S. F. P., Ottaviano E., Ceccarelli M., "A Characterization of the Workspace Boundary of Three-Revolute Manipulators", Design Engineering Technical Conferences (DETC-02), Proceedings of DETC-02, ASME 2002, Montreal, 1, 34342-34352, 2002.

[31] Storn R. M., Price K. V., Lampinen J. A., "Differential Evolution - A Practical Approach to Global Optimization”. Springer, Natural Computing Series, 2005.

[32] Storn R., Price K. V., "Differential Evolution: A Simple and Efficient Adaptive Scheme for Global Optimization Over Continuous Spaces”. International Computer Science Institute, 12, 1-16, 1995.

[33] Storn R., "Differential Evolution Design of an IIR-filter with Requirements for Magnitude and Group Delay", International Computer Science Institute, TR-95026, 1995.

[34] van Griensven A., Bauwens W., "Multiobjective Autocalibration for Semidistributed Water Quality Models", Water Resources Research, 39, 1348-1357, 2003, doi:10.1029/2003WR002284. 
[35] Vargas J. R. G., Villarreal L., Reynoso J. M., Mier-Maza R., "Diseo de un Manipulador Industrial para Aplicaciones de Limpieza en Subestaciones Elctricas". Centro Metropolitano de Investigacin en Mecatrnica, ITESM Quertaro, 1992. 